\title{
Gastric Bypass with Long Alimentary Limb or Long Pancreato-Biliary Limb-Long-Term Results on Weight Loss, Resolution of Co-morbidities and Metabolic Parameters
}

\author{
Bent Johnny Nergaard • Björn Geir Leifsson • \\ Jan Hedenbro • Hjörtur Gislason \\ Published online: 18 April 2014 \\ (C) The Author(s) 2014. This article is published with open access at Springerlink.com
}

\begin{abstract}
Background Several studies indicate that increasing the alimentary limb length in gastric bypass surgery produces only a minor improvement of excess BMI loss. Few studies have addressed the efficacy of increasing the length of the pancreatico-biliary limb.

Methods Here, we present a prospective randomized study of 187 consecutive laparoscopic Roux-Y gastric bypass operations operated over 2 years (2004-2005) in Iceland. The patients were operated with a gastric bypass with either a 2$\mathrm{m}$ biliopancreatic (BP)-limb and a 60-cm alimentary (A)-limb $(n=93)$ or with a $150-\mathrm{cm} \mathrm{A-limb}$ and a $60-\mathrm{cm} \mathrm{BP-limb}(n=$ 94).

Results Preoperative median BMI was 44.1 (38-70), median age 35.5 (17-74) years, and $85 \%$ of the patients were female. Follow-up rate after 5 years was $85 \%$. Eighteen months following surgery, the weight loss was significantly higher in the BP-limb group $(p<0.001)$, and this difference remained 7 years after surgery. Weight regain occurred in both groups, and 7 years after surgery, excess BMI loss (EBMIL) was $78.4 \%$ in the BP-limb group compared to $67.1 \%$ in the Alimb group $(p<0.001)$. Most patients $(78 \%)$ needed supplementation adjustment (iron, vitamin $\mathrm{D}$ and calcium) during the study period, significantly more often in the BP-limb group compared to the A-limb group $(p<0.001)$. Patients in the BP-limb group had more frequent stools than patients in
\end{abstract}

B. G. Leifsson · H. Gislason

Department of Surgery, Landspitali University Hospital, Reykjavik, Iceland

B. J. Nergaard • B. G. Leifsson · J. Hedenbro $\cdot$ H. Gislason $(\triangle)$ Department of Surgery, Aleris Hospital, Fredrik Stangs Gate 11-13, 0264 Oslo, Norway

e-mail: hjortur.gislason@aleris.no

B. J. Nergaard · B. G. Leifsson · J. Hedenbro · H. Gislason Aleris Obesity Skane, Kristianstad, Sweden the A-limb group; otherwise, gastro-intestinal symptoms rating scoring were comparable. Complication rate was similar. Conclusions Gastric bypass with a 2-m BP-limb gives better weight loss than gastric bypass with a $60-\mathrm{cm}$ BP-limb and a 150-cm A-limb. Metabolic follow-up is of utmost importance, as most patients needed repeated adjustments of their supplementation.

Keywords Bariatric surgery · RYGBP · Weight loss · Measured limbs · Gastric bypass

\section{Introduction}

Mason and Ito introduced gastric bypass (Roux-en-Y gastric bypass (RYGBP)) in the late 1960s, but still, there is no consensus on the ideal length of the gastric bypass limb lengths [1]. The operation results in excess weight loss (EWL) of 70-80\% after 1 to 2 years with high resolution of co-morbidities, but some patients regain significant weight, especially super obese patients. In order to improve results and to find the appropriate balance between benefits and side effects and metabolic sequels, studies with different limb lengths have been performed.

Many studies have been performed with variable lengths of the Roux limb (alimentary (A)-limb), ranging from 45 to $250 \mathrm{~cm}$; they show no or marginal difference in the effect on weight loss. Four of them are randomized studies [2-5], but most are retrospective comparisons [6-10]. Most of these studies are of small volume and not showing data beyond 5 years.

Many authors have demonstrated that weight loss after bariatric surgery and resolution of co-morbidities are strongly associated with a short common channel [11-15], but the shorter the common channel, the more malabsorption and metabolic problems can be expected $[7,12,14]$. 
However, it should also be noted that no reference can be made to the effect of the ratio of the Roux to the biliopancreatic (BP)-limbs on weight loss and co-morbidity resolution outcomes as there is no available literature that has investigated this issue [16]. Furthermore, long-term weight loss data ( $>5$ years) are not available from randomized controlled trials and are extremely limited in studies of other design [16]. Nevertheless, most authors believe that this ratio is unlikely to have any significant impact on outcome compared to the ratio of the Roux/BP to the common channel length.

The aim of this study was to compare the effect of the RYGBP with a 200-cm BP-limb (long BP) to a more commonly performed RYGBP with a $150-\mathrm{cm}$ long alimentary limb (150-cm A-limb) and a 60-cm BP-limb (long A) (Fig. 1). The long-term effects on weight loss, resolution of co-morbidities, gastro-intestinal symptoms, metabolic parameters and complications were examined.

\section{Materials and Methods}

\section{Patients}

Prior to this study, our standard laparoscopic gastric bypass procedure was Roux-en-Y gastric bypass with gastric pouch approximately $40 \mathrm{ml}$, biliopancreatic limb (BP-limb) of $200 \mathrm{~cm}$ and alimentary limb (A-limb) of $50 \mathrm{~cm}$ [17]. With this procedure, we had a problem with a high incidence of marginal ulcers (16.6 \% of patients) [17]. Six (comment 1 , reviewer 2) months before the study starts, we changed our procedure to a RYGBP with a gastric pouch of approximately $15 \mathrm{ml}$, BP-limb of $200 \mathrm{~cm}$ and A-limb of $60 \mathrm{~cm}$.

Between January 2004 and December 2005, all 187 morbidly obese patients who underwent primary RYGBP at our institution were included in the study. The same surgical team (HG, BGL) performed all operations. The patient selection criteria were $\mathrm{BMI} \geq 40$ or $\mathrm{BMI} \geq 35$ with associated comorbidity due to obesity (European Guidelines). Prior to surgery, patients participated in a multidisciplinary outpatients' preparation program, as described in detail earlier [17], and contracted to follow our follow-up program. A protocol was designed at the outset of the study for random allocation of the patients to either of the two arms. The surgical arms are illustrated in Fig. 1. Postoperatively, patients were instructed to take standard supplementation of vitamins and minerals including multivitamin, calcium citrate, vitamin B-12 and vitamin D. Patients were followed up at the outpatient clinic with weight registration and metabolic surveillance, first after 3 months and then every 6 months for 3 years, then once a year.

Resolution of diabetes type 2 was defined as patient without diabetic medication and with glycosylated haemoglobin $(\mathrm{HbAlc})$ within the normal range. Resolution of hypertension was defined as patient without treatment having blood pressure less than 140/90. Patients using continuous positive airway pressure (CPAP) were considered to have sleep apnoea.

Six to 7 years after surgery, patients received a standardized questionnaire including gastro-intestinal symptom rating scale (GSRS). It is an interview-based rating scale consisting of 15 items, originally constructed for the assessment of gastrointestinal symptoms in the irritable bowel syndrome and
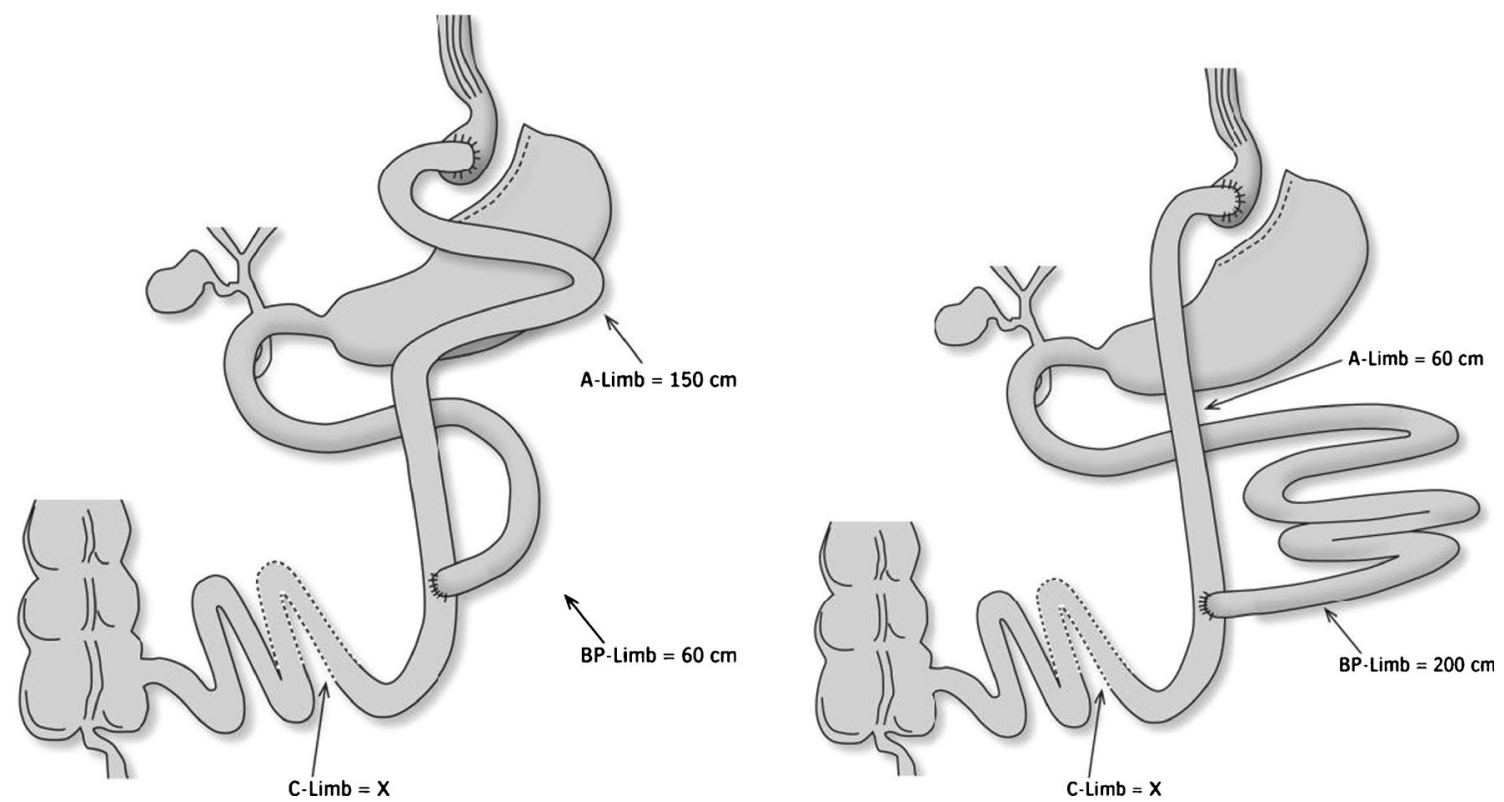

Fig. 1 Schematic drawing of the operation with 150-cm A-limb or 200-cm BP-limb 
Table 1 Patient demographics

\begin{tabular}{llll}
\hline & $\begin{array}{l}\text { Long BP-limb } \\
(n=94)\end{array}$ & $\begin{array}{l}\text { Long A-limb } \\
(n=93)\end{array}$ & $p$ Value \\
\hline Age (years) & $34(17-74)$ & $37(22-61)$ & 0.383 \\
Gender, F/M & $79 / 15(84 \%)$ & $81 / 12(87 \%)$ & 0.678 \\
BMI & $44.5(39-70)$ & $43.7(38-68)$ & 0.595 \\
Super obese (BMI $>50)$ & $35(37.2 \%)$ & $31(33.3 \%)$ & 0.647 \\
Weight $(\mathrm{kg})$ & $139(115-225)$ & $134(92-215)$ & 0.141 \\
\hline
\end{tabular}

Values given are median (range)

or $\%$

Comparison of mean percentage excess BMI loss (EBMIL\%) between groups was done using Student's $t$ test. A $p$ value $<0.05$ was regarded as statistically significant. Weight loss was expressed as percentage excess BMI loss (EBMIL\%) plotted against time from operation. The lines were drawn using the locally weighted scatter plot smoothing method (Lowess). All statistical computations were performed using the SPSS version 21.0 for MacOS (SPSS Inc., Chicago).

\section{Statistics}

Data from all the patients during the study period were prospectively collected in a proprietary database as part of the hospital records. Proportions are referred to as numbers (\%). Continuous data are presented as median and range unless otherwise stated. Differences between proportions were evaluated using Fisher's exact test. Median differences between groups were evaluated using the Mann-Whitney $U$ test.

\section{Surgical Procedure}

Five ports were used: two $5-\mathrm{mm}$ and two $12-\mathrm{mm}$ ports for instruments and one $10-\mathrm{mm}$ port for the camera. A small gastric pouch $(15 \mathrm{ml})$ was created with a $3.5-\mathrm{mm}$ stapler (Endo-GIA II, Tyco/US Surgical). The greater omentum was then split vertically to allow a tension-free antecolic gastrojejunal anastomosis (GJ anastomosis). The ligament of Treitz

Fig. 2 Postoperative weight loss expressed as percentage of excess BMI loss (EBMIL\%) plotted against time. The lines are drawn using the locally weighted scatter plot smoothing method (Lowess). The figures demonstrate a greater weight loss using the long BPlimb procedure compared to long A-limb procedure

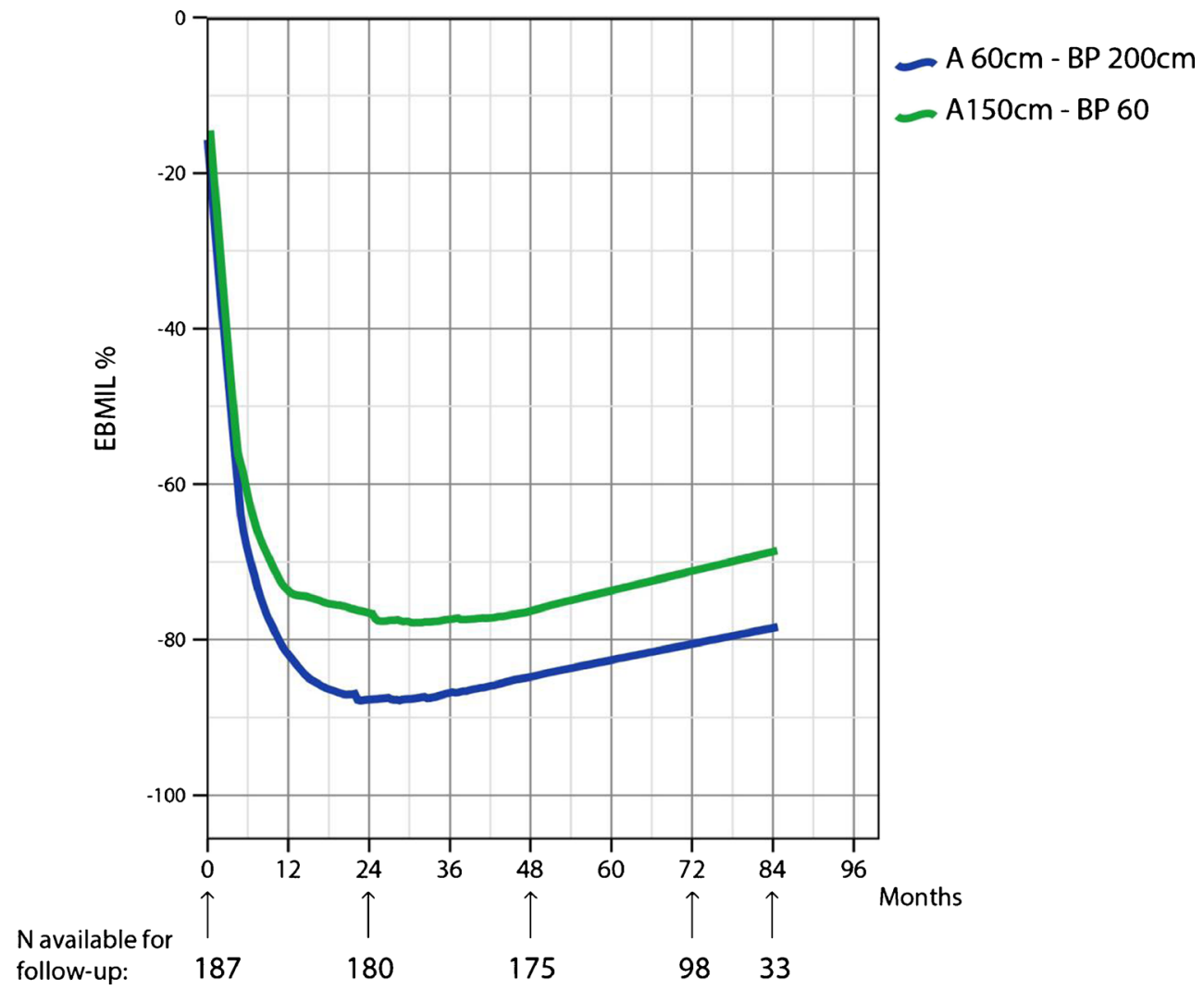


was identified, and jejunum measured (loose, not stretched using markers on the graspers) (comment 2, reviewer 2) 60 or $200 \mathrm{~cm}$ according to the group included (long A or long BP). The bowel was brought up, first as an omega loop in an antecolic and antegastric fashion, and the GJ anastomosis was created by stapling the jejunum to the posterior wall of the gastric pouch using a linear stapler (3.5-mm stapler). The entero-entero anastomosis (EE anastomosis) was created as a side-to-side anastomosis either 60 or $150 \mathrm{~cm}$ below the GJ anastomosis using a $2.5-\mathrm{mm}$ stapler. The remaining openings were hand-sutured and the omega loop divided by the $2.5-\mathrm{mm}$ stapler. The last step was testing the integrity of the gastrojejunostomy by inflating it with methylene-blue-dyed saline via an NG tube. The mesenteric defects were not closed. Our surgical procedure at the time has previously been described in detail in 2005 [17, 22].

\section{Results}

The results of 187 consecutive patients equally distributed (2-3 patients weekly) over a 2-year period (2004-2005) are presented. Laparoscopic surgery was successfully completed in all cases. Ninety-four patients were randomized to the BP-limb group and 93 patients to the A-limb group. Patient's characteristics in the two groups are shown in Table 1.

A total of $85.6 \%$ of patients $(160 / 187)$ were females, median age 35.5 (17-74). Before the start of the treatment, median weight was $136.5 \mathrm{~kg}(92-225)$ and median BMI was 44.1 (38.3-70.3). Sixty-six (35.3\%) patients were super obese $(\mathrm{BMI}>50)$. Mean weight loss prior to surgery was $12.7 \mathrm{~kg}$ $(0-46)$, similar in both groups. The median surgical time was $67.3 \mathrm{~min}(37-112), 68.3 \mathrm{~min}(40-112)$ in the BP-limb group compared to $66.3 \mathrm{~min}(37-94)$ in the A-limb group $(p=0.29)$ (comment 9, reviewer 2). The median hospital stay was 2.9 days (2-10 days), 2.7 days (2-6) in the BP-limb group and 3.1 days $(1-10)$ in the A-limb group $(p=0.30)$ (comment 9 , reviewer 2). There was no difference between the two groups (Table 1).

The diameter of small intestine just below the gastrointestinal anastomosis was measured in 20 patients in each group with a soft plastic measuring tape (comment 3, reviewer 2). In the A-limb group, the median diameter was $3.5 \mathrm{~cm}(3.0-4.3)$ compared to $2.3 \mathrm{~cm}(1.9-3.1)$ in the BPlimb group $(p<0.05)$.

The median follow-up was 70.6 months (0.4-93), similar in both groups. As shown in Fig. 2, 159 patients (85.0 \%) attended our outpatient clinic for follow-up of 5 years, and 110
Fig. 3 Postoperative weight loss expressed as percentage of excess BMI loss (EBMIL\%) plotted against time. The figures demonstrate a greater weight loss in both obese and super obese patients using the long BP-limb procedure compared to long Alimb procedure

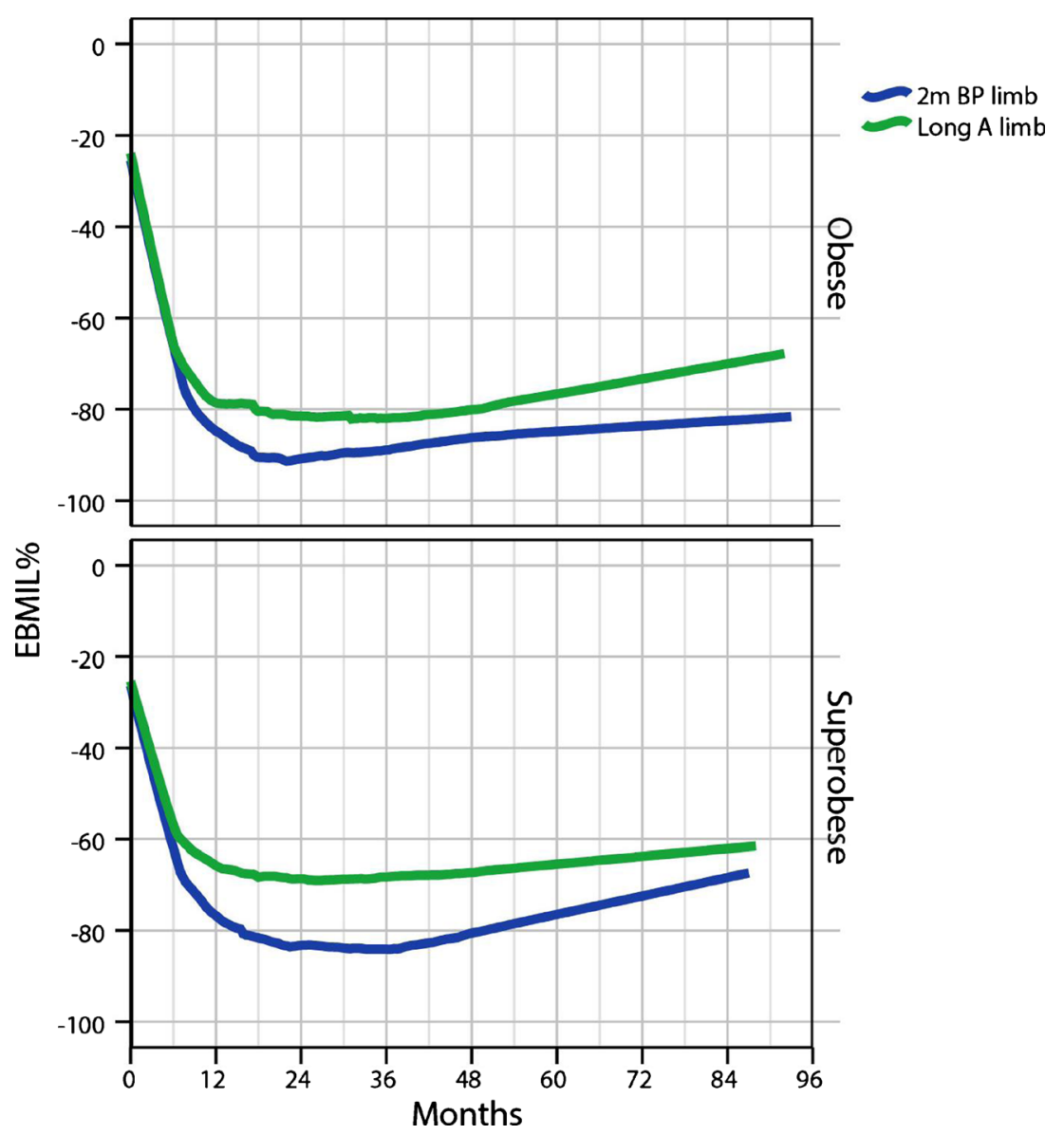


Table 2 Late complications (>30 days)

or $\%$

\begin{tabular}{lllll}
\hline & $\begin{array}{l}\text { All patients } \\
(n=187)\end{array}$ & $\begin{array}{l}\text { Long BP-limb } \\
(n=94)\end{array}$ & $\begin{array}{l}\text { Long A-limb } \\
(n=93)\end{array}$ & $p$ Value \\
\hline Internal hernia & $15(8 \%)$ & $9(10 \%)$ & $6(6 \%)$ & 0.592 \\
Marginal ulcer & $16(8.6 \%)$ & $8(9 \%)$ & $8(9 \%)$ & 1.000 \\
Gallbladder stones & $22(11.8 \%)$ & $13(14 \%)$ & $9(10 \%)$ & 0.497 \\
\hline
\end{tabular}

(59\%) completed follow-up 6 to 7 years after surgery. Twenty-eight patients $(15 \%)$ had a follow-up of less than 5 years including three patients who died 1, 2 and 4 years after surgery of unrelated causes.

Figure 2 shows the percentage excess BMI loss (EBMIL\%) in the two groups. For 18 months, weight loss was significantly higher in the BP-limb group $(p<0.001)$, and this difference remained throughout the study period. Mean excess BMI loss reached a maximum of $88.5 \%$ in the BP-limb group 2 years after surgery compared to $77.7 \%$ in the A-limb group. Weight regain occurred in both groups, and 7 years after surgery, mean EBMIL was $78.4 \%$ in the BP-limb group compared to $67.1 \%$ in the A-limb group. Weight loss failure defined as EBMIL\% never exceeding $50 \%$ postoperatively was only seen in four patients in the A-limb group compared to none in the 2-m BP-limb group.

Figure 3 (upper part) shows the EBMIL\% in those 110 study patients who were not super obese $(\mathrm{BMI} \leq 50)$. Seven years after surgery, mean EBMIL was $82.2 \%$ for the BP-limb group compared to $69.2 \%$ for the A-limb group $(p<0.001)$.
Figure 3 (lower part) shows, however, EBMIL\% in 66 preoperatively super obese patients $(\mathrm{BMI}>50)$. Seven years after surgery, mean EBMIL was $70.4 \%$ in the BP-limb group compared to $62.8 \%$ in the A-limb group ( $p<0.01$ ).

Early complications ( $<30$ days) did not differ between the two groups. Two patients had leaks (1.1\%) treated by surgery; five patients $(2.7 \%)$ had bleedings, two treated by surgery and three conservatively.

Late complications ( $>30$ days) are shown in Table 2. Seven to 9 years after gastric bypass, 15 patients $(8 \%)$ had surgery due to internal hernia, 9 in the BP-limb group and 6 in the Alimb group ( $p=0.592)$. Sixteen patients $(8.6 \%)$ had marginal ulcers at the gastro-jejunal anastomosis treated conservatively, eight in each group. Twenty-two patients (11.8\%) had surgery due to gallbladder stones (preoperative ultrasound was not performed) (comment 6 , reviewer 2).

Co-morbidity was similar in both groups prior to surgery (comment 9, reviewer 2). Table 3 shows the resolution of obesity-related co-morbidities 5 to 9 years after gastric bypass. A total of $20.9 \%(39 / 187)$ were treated for diabetes type 2

Table 3 Preoperative incidence of obesity-related co-morbidities and the changes 5 to 9 years after Roux-en-y gastric bypass

\begin{tabular}{|c|c|c|c|c|c|}
\hline & All patients $(n=187)$ & Study groups & Preoperative incidence (\%) & Resolved (\%) & $p$ Value \\
\hline \multirow[t]{2}{*}{ Diabetes type 2} & $39(20.9 \%)$ & Long BP-limb $(n=94)$ & $22(23)$ & $17(77)$ & 0.636 \\
\hline & & Long A-limb $(n=93)$ & $17(18)$ & $12(71)$ & \\
\hline \multirow[t]{2}{*}{ Hypertension } & $59(31.6 \%)$ & Long BP-limb $(n=94)$ & $28(30)$ & $17(61)$ & 0.406 \\
\hline & & Long A-limb $(n=93)$ & $31(33)$ & $22(71)$ & \\
\hline \multirow[t]{2}{*}{ Sleep apnoea } & $31(17 \%)$ & Long BP-limb $(n=94)$ & $19(20)$ & $17(90)$ & 0.245 \\
\hline & & Long A-limb $(n=93)$ & $12(13)$ & $12(100)$ & \\
\hline \multicolumn{6}{|l|}{ B } \\
\hline & & All patients $(\%)$ & Long BP-limb (\%) & Long A-limb (\%) & $p$ Value \\
\hline \multirow[t]{5}{*}{ GERD } & Worse & 17 & 8 & 21 & \\
\hline & No change & $4 \%$ & 0 & 10 & \\
\hline & Slightly improved & 8 & 8 & 10 & \\
\hline & Much improved & 8 & 0 & 17 & \\
\hline & Resolved & 63 & 84 & 42 & 0.140 \\
\hline \multirow[t]{5}{*}{ Joint pain } & Worse & 19 & 25 & 16 & \\
\hline & No change & 29 & 10 & 41 & \\
\hline & Slightly improved & 15 & 25 & 9 & \\
\hline & Much improved & 29 & 35 & 25 & \\
\hline & Resolved & 8 & 5 & 9 & 0.136 \\
\hline
\end{tabular}

Values given are number of patients and \%; statistic calculated (Chi-square) in each group is based on no improvement versus improvement 
Table 4 Nutritional parameters outside reference values, number of patients (\%)
Values given are number of patients and $\% ; p$ values indicate difference in prevalence between the two arms of the study; $p<0.05$ is taken to indicate statistical significance

\begin{tabular}{lllll}
\hline & Before surgery & \multicolumn{2}{l}{$\begin{array}{l}3 \text { to } 9 \text { years after surgery }(n=177), \text { median } \\
\text { follow-up 71.8 months }(36-93)\end{array}$} \\
\cline { 3 - 5 } & $\begin{array}{l}\text { All patients } \\
(n=187)\end{array}$ & $\begin{array}{l}\text { Long BP-limb } \\
(n=87)\end{array}$ & $\begin{array}{l}\text { Long A-limb } \\
(n=86)\end{array}$ & $p$ Value \\
\hline Albumin & $7(3.7 \%)$ & $23(26.4 \%)$ & $18(20.9 \%)$ & 0.394 \\
Vitamin B-12 & $12(6.4 \%)$ & $24(26.4 \%)$ & $16(18.6 \%)$ & 0.161 \\
Vitamin D & $57(30.5 \%)$ & $39(44.8 \%)$ & $18(20.9 \%)$ & $<0.001$ \\
PTH & $1(0.5 \%)$ & $15(17.2 \%)$ & $3(3.5 \%)$ & 0.003 \\
Iron & $2(6.4 \%)$ & $32(36.8 \%)$ & $13(15.1 \%)$ & $<0.001$ \\
Ferritin & $11(5.9 \%)$ & $58(66.7 \%)$ & $45(52.3 \%)$ & 0.055 \\
Haemoglobin & $2(1.1 \%)$ & $53(60.9 \%)$ & $37(43.0 \%)$ & 0.019 \\
Iron and ferritin & $0(0 \%)$ & $20(23.0 \%)$ & $12(14.0 \%)$ & 0.126 \\
Iron and ferritin and haemoglobin & $0(0 \%)$ & $15(17.2 \%)$ & $10(11.6 \%)$ & 0.185 \\
\hline
\end{tabular}

before surgery, 30 with oral antidiabetic medication only and 9 with insulin (comment 7, reviewer 2). Five to 9 years after surgery, 29 (74\%) had resolution of diabetes, no difference between the two study groups. Fifty-nine patients (31\%) were on treatment for hypertension; 39 patients $(66 \%)$ had resolution, no difference between the two surgical groups. Prior to surgery, 19 patients had sleep apnoea using CPAP; after 5 to 9 years, $93 \%(17 / 19)$ were not in need of CPAP. Thirteen patients $(6.9 \%)$ had hyperlipidaemia prior to surgery; all of them had a resolution 5 years or more after surgery (comment 10 , reviewer 2). Before surgery, 37 patients (19.8\%) had GERD (18 patients in the BP-limb and 19 in the A-limb group) (comment 9, reviewer 2). Joint pain was recorded in 105 patients $(56.1 \%)$ prior to surgery (comment 10 , reviewer 2). Dramatic improvement of GERD and joint pain was registered in most patients after surgery, similar in both study groups (Table 3).

Table 4 shows the nutritional parameters in all patients prior to surgery. A total of $35.5 \%$ of the patients had nutritional deficiency before surgery, most commonly vitamin D deficiency $(31 \%)$. This was corrected postoperatively by adding supplementation as needed in addition to the standard supplementation.

Table 4 shows the percentage of patients in the two groups needing adjustment of supplementation because of their laboratory values being outside the reference values, three or more years after surgery. Most patients (78\%) needed frequent adjustment of their supplementation during the followup period of 3 to 9 years. Need for adjustment of iron, vitamin $\mathrm{D}$ and calcium citrate was significantly more common in the BP-limb group compared to the A-limb group $(p<0.001)$. Only one patient (long BP) needed hospitalization due to significant but reversible malnutrition problems caused by serious eating disorder. Otherwise, nutrition problems were solved at the outpatient clinic.

A total of 105 patients (56\%) answered the questionnaire. The GSRS scores represented gastro-intestinal symptoms and associated complaints. Higher score represents more symptoms. As shown in Table 5, the diarrhoea score representing frequency of stools, loose stools and urgency was significantly higher in the BP-limb group compared to the A-limb group. The total GSRS score in the BP-limb group was, however, not significantly different from that in the A-limb group (11.1 vs 9.81).

\section{Discussion}

The fact that bariatric surgery can fall short of patients' expectations is troublesome. Both the amount of initial weight loss and the risk of later weight regain are issues that need
Table 5 Assessment of symptoms by the gastro-intestinal symptom rating scale (GSRS)

Values given are mean (SD); $p$ values indicate difference in prevalence between the two arms of the study

\begin{tabular}{llll}
\hline & $\begin{array}{l}\text { Long BP-limb } \\
(n=94)\end{array}$ & $\begin{array}{l}\text { Long A-limb } \\
(n=93)\end{array}$ & $p$ Value \\
\hline GSRS abdominal pain & $2.13(n=48)$ & $2.01(n=57)$ & 0.60 \\
GSRS constipation & $2.09(n=48)$ & $2.13(n=57)$ & 0.90 \\
GSRS diarrhoea & $2.66(n=48)$ & $1.19(n=57)$ & 0.007 \\
GSRS indigestion & $2.76(n=48)$ & $2.36(n=57)$ & 0.14 \\
GSRS reflux & $1.45(n=48)$ & $1.40(n=57)$ & 0.83 \\
Total GSRS score & 11.10 & 9.81 & \\
\hline
\end{tabular}


addressing. The mechanisms behind the effect of gastric bypass are not yet fully understood. We chose to study the effect of different limb lengths in a group of consecutive patients, including both morbidly and super obese.

We found that the longer BP-limb patients did better in terms of weight loss and showed less weight regain over a 7year period. This effect was achieved at the price of more GI symptoms and a higher need of supplementation.

Why does the 200-cm BP-limb RYGPB results in greater weight loss than the $150-\mathrm{cm}$ A-limb? When performing gastric bypass with a $200-\mathrm{cm}$ BP-limb, the whole jejunum (in most patients) is bypassed and the upper anastomosis is in fact a gastro-ileal anastomosis. The proposed mechanisms could be the following: (a) Food directly to the ileum could affect food tolerance and thereby eating behaviour, and (b) by creating a $200-\mathrm{cm}$ BP-limb, most of the foregut is bypassed altering more, or differently, hormonal and immunological factors. We think the main mechanism is probably the different profile of the GI hormones as demonstrated by numerous recent studies on metabolic surgery (comment 8 , reviewer 2). (c) In the 200-cm BP-limb group, $50 \mathrm{~cm}$ more of the intestine is bypassed creating consequently a shorter common channel.

In our database, we have measured the total small intestinal length in 650 patients to be $620 \mathrm{~cm}(420-870 \mathrm{~cm})$ (unpublished results). Thus, the common channel in our study will be more than $3 \mathrm{~m}$ in most patients. There is convincing evidence that the degree of malabsorption after gastric bypass is influenced mainly by the length of the common channel rather than the lengths of the Roux or biliopancreatic limbs as bypass is currently constructed by the majority of bariatric surgeons [16]. Thus, the common channel in our study is probably long enough to ensure full uptake of calories, so malabsorption of calories will not explain the difference in weight loss between the two groups. The tendency of the two curves to converge in the super obese group should be interpreted with caution as this is based on few data points. There may also be a follow-up bias favouring those with weight regain problems. Further, follow-up will reveal whether this tendency will hold.

The metabolic problems associated with biliopancreatic diversion and very long limb RYGBP are mostly due to the short common limb [11-15, 23].

We found that 2-m BP-limb is associated with more micronutrient deficiency than $60-\mathrm{cm}$ BP-limb and $150-\mathrm{cm}$ long A-limb, especially iron and calcium deficiency, but these elements are known to be primarily absorbed from the proximal part of the intestines. However, most patients in both groups needed frequent adjustments of their supplementation also 5 years or more after surgery, emphasizing the need for lifelong follow-up after gastric bypass.

Six to 7 years after surgery, gastro-intestinal symptoms were similar in both study groups; the only significant difference was more frequent and looser stools in the BP-limb group. Gastro-intestinal symptoms usually diminish or disappear over time, and differences in symptoms between groups may have been more pronounced earlier after surgery.

Before this study (in our learning curve), we performed a gastric bypass with a 2-m BP-limb and a large gastric pouch $(50 \mathrm{ml})$ [17]; this resulted in high ulcer rate $(17 \%)$; the pouch could dilate and many patient had GI symptoms. By reducing the pouch to $15 \mathrm{ml}$ and taking good care of the vascularisation of the major curvature flap, the ulcer rate dropped to $9 \%$ (same in between groups) and the GI symptoms diminished.

Differences in technique other than limb length (such as size of gastric pouch or stoma size) may account for weight loss differences and make it difficult to compare weight loss results between different studies.

Lee et al. [24] performed loop (mini) gastric bypass with the BP-limb tailored according to patients' BMI from 150 to $350 \mathrm{~cm}$ and describe a linear relationship between the reduction in BMI and the BP-limb length. In accordance to our results, the same group found that a routine use of 2-m BPlimb mini bypass not only increased weight loss but also increased the incidence of late nutritional deficiencies, especially anaemia [25].

We conclude that a gastric bypass with a 2-m BP-limb gives a better long-term weight loss than gastric bypass with a $150-\mathrm{cm}$ A-limb, with similar complication rate, but with more nutritional deficiency. This difference is unlikely due to more malabsorption but more likely caused by other mechanisms.

Conflict of Interest All authors declare that they have no conflict of interest and the study has not been supported by any grants.

Open Access This article is distributed under the terms of the Creative Commons Attribution License which permits any use, distribution, and reproduction in any medium, provided the original author(s) and the source are credited.

\section{References}

1. Mason EE, Ito C. Gastric bypass. Ann Surg. 1969;170:329-39.

2. Brolin RE, Kenler HA, Gorman JH. Long-limb gastric bypass in the super obese. A prospective randomized study. Ann Surg. 1992;215: 387-95.

3. Choban PS, Flancbaum L. The effect of Roux limb lengths on outcome after Roux-en-Y gastric bypass: a prospective, randomized clinical trial. Obes Surg. 2002;12:540-5.

4. Inabnet WB, Quinn T, Gagner M, et al. Laparoscopic Roux-en-Y gastric bypass in patients with $\mathrm{BMI}<50$ : a prospective randomized trial comparing short and long limb lengths. Obes Surg. 2005;15:51-7.

5. Pinheiro JS, Schiavon CA, Pereira PB. Long-long limb Roux-en-Y gastric bypass is more efficacious in treatment of type 2 diabetes and lipid disorders in super-obese patients. Surg Obes Relat Dis. 2008;4:521-7.

6. Freeman JB, Kotlarewsky M, Phoenix C. Weight loss after extended gastric bypass. Obes Surg. 1997;7:337-44.

7. Brolin RE, LaMarca LB, Kelner HA, et al. Malabsorptive gastric bypass in patients with superobesity. J Gastrointest Surgery. 2002;6: 195-205. 
8. Feng JJ, Gagner M, Pomp A, et al. Effect of standard vs extended Roux limb length on weight loss outcomes after laparoscopic Rouxen-Y gastric bypass. SurgEndosc. 2003;17:1055-60.

9. Christou NV, Look D, MacLean LD. Weight gain after short- and long-limb gastric bypass followed for longer than 10 years. Ann Surg. 2006;244:734- 40 .

10. Ciovica R, Takata M, Vittinghoff E, et al. The impact of roux limb lengths and weight loss after gastric bypass. Obes Surg. 2008;18:510 .

11. Scopinario N, Gianetta E, Civalleri D, et al. Bilio-pancreatic bypass for obesity: initial experience in man. Br J Surg. 1979;66(9):618-20.

12. Sugerman HJ, Kellum JM, DeMaria EJ. Convertion of proximal to distal bypass for failed gastric bypass for superobesity. J Gastrointest Surg. 1997;1:517-26.

13. Brolin RE, Cody RP. Adding malabsorption for weight loss failure after gastric bypass. SurgEndosc. 2007;21:1924-6.

14. Nelson WK, Fatima J, Houghton SG, et al. The malabsorptive very, very long limb Roux-en-Y gastric bypass for super obesity: results in 257 patients. Surgery. 2006;140:517-23.

15. McConnell DB, O'Rourke RW, Deveney CW. Common channel length predicts outcomes of biliopancreaticdivertion alone and with the duodenal switch surgery. Am J Surg. 2005;189:536-40.

16. Dimitrios S, Kuwada TS, Gersin KS. The importance of the length of the limbs for gastric bypass patients - an evidence-based review. ObesSurg. 2011;21:119-24.

17. Leifsson BG, Gislason HG. Laparoscopic Roux-en-Y gastric bypass with 2-metre long biliopancreatic limb for morbid obesity: Technique and experience with the first 150 patients. ObesSurg. 2005;15(1):3542.

18. Dimenäs E, Glise H, Hallerbäck B, et al. Well-being and gastrointestinal symptoms among patients referred to endoscopi owing to suspected duodenal ulcer. Scand J Gastroenterol. 1995;30:1046-52.

19. Svedlund J, Sjödin I, Dotevall G. GSRS - A clinical rating scale for gastrointestinal symptoms in patients with irritable bowel syndrome and peptic ulcer disease. Dig Dis Sci. 1988;33:129-34.

20. Svedlund J, Sullivan M, Liedman B, et al. Long term consequences of gastrectomy for patients quality of life: the impact of reconstructive techniques. Am J Gastroenterol. 1999;94:438-45.

21. Wiklund I, Carlsson J, Vakil N. Gastroesophageal reflux symptoms and well-being in a random sample of the general population of a Swedish community. Am J Gastroenterol. 2006;101:18-28.

22. Jacobsen HJ, Bergland A, Raeder J, Gislason HG. High-volume bariatric surgery in a single centre: Safety, quality, cost-efficacy and teaching aspects in 2,000 consecutive cases. ObesSurg. 2012;22: $158-66$.

23. Scopinaro N, Gianetta E, Adami GF, et al. Biliopancreatic diversion for obesity at eighteen years. Surgery. 1996;119:261-8.

24. Lee WJ, Wang W, Lee YC, et al. Laparoscopic mini gastric bypass: experience with tailored bypass limb according to body weight. ObesSurg. 2008;3:294-9.

25. Lee WJ, Yu PJ, Wang W, et al. Laparoscopic Roux-en-Y versus minigastric bypass for the treatment of morbid obesity. Ann Surg. 2005;242:20-8. 